\title{
B-WIM System Using Fewer Sensors
}

\section{Yahya M. Mohammed ${ }^{1}$, Nasim Uddin ${ }^{2}$}

${ }^{1}$ Ph.D. student, Research Assistant, Department of Civil Construction and Environmental Engineering, University of Alabama at Birmingham, Birmingham, AL, 35294, USA. Email: yahya1@uab.edu.

${ }^{2}$ Ph.D., P.E., Professor, Department of Civil Construction and Environmental Engineering, University of Alabama at Birmingham, Birmingham, AL, 35294, USA.Email: nuddin@uab.edu.

\begin{abstract}
Bridge Weigh-in-Motion (B-WIM) is the concept of using measured response on a bridge to calculate the static weights of passing traffic loads as they pass overhead at full highway speed. This paper describes an enhancement to the Moving Force Identification (MFI) algorithm by estimating the response of some DOFs using limited number of measurements in order to increase measurements number (Input). The pseudoinverse of the mode shape matrix has been utilized to approximately calculate the modal response using limited measured response. Then the calculated modal response has been used to estimate more DOFs that are different from the measured one. The proper orthogonal decomposition (POD) technique is employed to determine the governing modes that increase the modal response accuracy. Numerical example for quarter car model passing over simply supported bridge has been established to demonstrate the idea.

Keywords: Moving Force Identification (MFI); Bridge Weigh in Motion (B-WIM); Truck weight; Inverse Dynamics; Pseudoinverse; Proper Orthogonal Decomposition

\section{Introduction}

The American Society of Civil Engineers (ASCE) reported, in the Report Card for America' s Infrastructure, that almost four in each 10 bridges are 50 years or older. 56,007 - 9.1\% - of America' s bridges were structurally deficient in 2016, and on average there were 188 million trips across a structurally deficient bridge each day ${ }^{[1]}$. In many cases, the current loading on these bridges is significantly different from the service loads at the time of design and construction. To facilitate bridge safety assessment, techniques have been developed to calculate the weights of trucks using the transportation network. Static weighing at weigh stations is accurate but can only provide data for a small sample of passing vehicles ${ }^{[2]}$. Weigh-in-Motion (WIM) is less accurate but can provide weights for all passing trucks. Bridge Weigh-in-Motion (B-WIM) is one of a number of WIM technologies and accuracies have been reported to be similar to those from other technologies ${ }^{[3]}$.

B-WIM systems have the potential to provide an inexpensive portable method of rapidly assembling traffic weight data. The novel technique of using the bridge as an instrument to infer the dynamic wheel forces was first investigated by ${ }^{[4]}$. Since then the field of moving force identification (MFI) has progressed rapidly ${ }^{[5-19]}$. The MFI problem is an inverse dynamics process and like many, is ill-conditioned which means the solution is very sensitive to small perturbations in measurements, which requires the regularization approach to overcome this problem. Law and Fang ${ }^{[7]}$ apply a dynamic programming technique to the MFI problem on a simply supported beam model using zeroth-order regularization to solve the state space formulation, then González ${ }^{[20]}$ extend the algorithm to allow for the first order regularization of moving forces, which improved the solution accuracy. Recently the MFI technique has been used to detect the damage location ${ }^{[21]}$. The accuracy of the MFI algorithm depends on the measurement numbers, which increases when using more sensors and decreases when utilizing less number of sensors. Also, the instrumentation cost and the installation time especially for high elevation bridges are very expensive which limit the use of the B-WIM technique.

Copyright (C) 2018 Yahya M. Mohammed et al.

doi: 10.24294/tm.v1i2.701

EnPress Publisher LLC.This work is licensed under the Creative Commons Attribution-NonCommercial 4.0 International License (CC BY-NC 4.0).

http://creativecommons.org/licenses/ by/4.0
\end{abstract}


This paper investigates an estimation technique, which allows the use of small number of sensors to operate the B-WIM system and increase the system accuracy at the same time. The estimation process is summarized as follows:

- Converting limited measured nodal structural responses to modal ones using the pseudoinverse of the mode shape matrix.

- $\quad$ Estimate more structural response using mode shape matrix.

- Apply MFI algorithm using the measured and the estimated response.

\section{Moving Force Identification (MFI) Algorithm}

The MFI algorithm uses inverse dynamics theory to back-calculate a complete time force history for axles or wheels that move on the bridge. The algorithm adopted in this paper is that used by González et al. ${ }^{[20]}$ who improve the work of Law et al. ${ }^{[7]}$ by applying the first-order regularization technique.

\section{Estimated Response}

Based on the pseudoinverse inverse of the mode shape matrix $\Phi^{+}$, the modal displacement responses $(U)$ can be approximately calculated from limited measured displacement responses $(y)$, and then the modal displacement responses can be utilized to back estimate another displacement response at different location as follows:

$$
\begin{gathered}
U_{q x 1}=\left(\Phi_{p x q}\right)^{+} y_{p x 1} \\
y_{p^{\prime} \mathrm{x} 1}=\Phi_{p^{\prime} x q} U_{q x 1}
\end{gathered}
$$

Where $U$ and $y$ are the modal and the measured displacement response respectively, $p$ donates the number of measurements, $q$ is the number of modes and $p^{\prime}$ is the number of estimated displacement. The error between the exact and estimated modal displacement responses can be minimized by choosing the number of sensors exceeding the number of modes governing the structural responses.

To determine the governing modes of a multi-degrees of freedom (MDOF) system, the proper orthogonal decomposition (POD) technique is employed ${ }^{[22]}$. The energy contribution $\theta$ of the first $q$ vibration modes can be calculated based on the POD method as

$$
\theta=\frac{\sum_{i}^{q} \lambda_{i}}{\sum_{i}^{n} \lambda_{i}}(q \leq n)
$$

Where $\lambda_{i}$ is the ith eigenvalue of the covariance matrix of the acceleration response, which represents the energy contribution to the response. The required energy contribution of the selected governing modes should exceed $90 \%$ of the total energy of the structural response in order to obtain an accurate estimation for the displacement response ${ }^{[23]}$.

\section{Numerical Simulation}

The vehicle is modeled as a quarter-car model crossing a 20-m approach distance followed by a 10-m simply supported finite element (FE) bridge (Figure 1). The quarter-car travels with constant speed $10 \mathrm{~m} / \mathrm{s}$. The vehicle masses are represented by a sprung mass, $\mathrm{m}_{\mathrm{s}}$, and un-sprung mass, $\mathrm{m}_{\mathrm{a}}$ represents the vehicle axle mass and body mass respectively. The Degrees of Freedoms (DOFs) that correspond to the bouncing of the sprung and the axle masses are, $\mathrm{u}_{\mathrm{s}}$, and $\mathrm{u}_{\mathrm{a}}$, respectively. The bridge is modeled with 1D Euler - Bernoulli finite beam elements with two degrees of freedom per node, vertical translation, and rotation. The properties of the quarter-car and the bridge are listed in Table 1 and based upon the work of Cebon ${ }^{[24]}$ and Harris, OBrien and González ${ }^{[25]}$. The road surface profile is not considered in this simulation. The dynamic interaction between the vehicle and the bridge is implemented in MATLAB ${ }^{[10,26]}$ based on the contact force concept adopted by Yang et $a l^{[27]}$. and Gonzalez ${ }^{[28]}$. Unless otherwise mentioned, the used scanning frequency is $1000 \mathrm{~Hz}$.

The bridge has been divided into to 10 equal elements and the displacement has been extracted at two different locations (4.0m, and 5.0m) away from the left support (Figure 2-a). Firstly, the pseudoinverse of the mode shape matrix has been used to transform the measured displacement to the modal one. Then, the modal displacement response has been utilized to estimate the remaining displacement at the others locations $(3.0,6.0,7.0,8.0) \mathrm{m}$ away from the left support (Figure 2-b). The figure shows that the pseudoinverse of the mode shape can estimate the bridge response 
(displacement in this example) with high accuracy and enhancing the force estimation process (Figure 3).

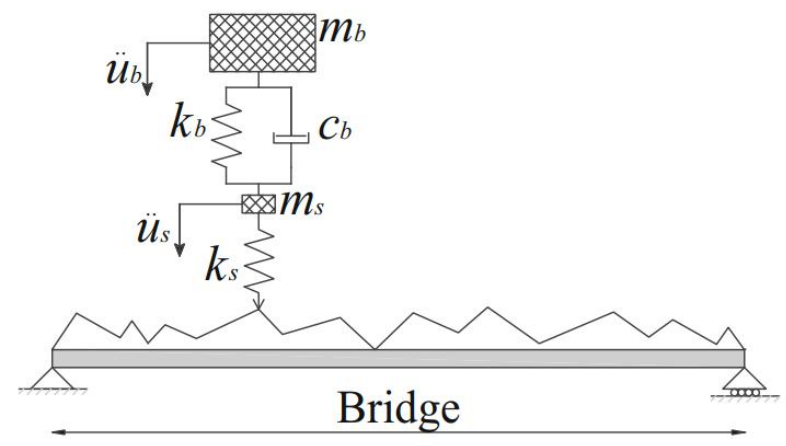

Figure 1; Theoretical quarter car model on Simply supported beam.

\begin{tabular}{|l|l|l|l|}
\hline \multicolumn{2}{|l|}{ Vehicle properties } & \multicolumn{3}{l|}{ Bridge properties } \\
\hline $\mathrm{mb}$ & $17300 \mathrm{~kg}$ & Span & $10 \mathrm{~m}$ \\
\hline $\mathrm{ms}$ & $700 \mathrm{~kg}$ & Density & $2400 \mathrm{~kg} / \mathrm{m}^{3}$ \\
\hline $\mathrm{kb}$ & $4 \mathrm{e} 5 \mathrm{~N} / \mathrm{m}$ & Width & $4.04 \mathrm{~m}$ \\
\hline $\mathrm{cb}$ & $1 \mathrm{e} 4 \mathrm{~N} \mathrm{~s} / \mathrm{m}$ & Depth & $0.51 \mathrm{~m}$ \\
\hline $\mathrm{K}_{\mathrm{s}}$ & $1.75 \mathrm{e} 6 \mathrm{~N} / \mathrm{m}$ & Modulus & $3.5 \mathrm{e} 10 \mathrm{~N} / \mathrm{m}^{2}$ \\
\hline
\end{tabular}

Table 1. Bridge properties

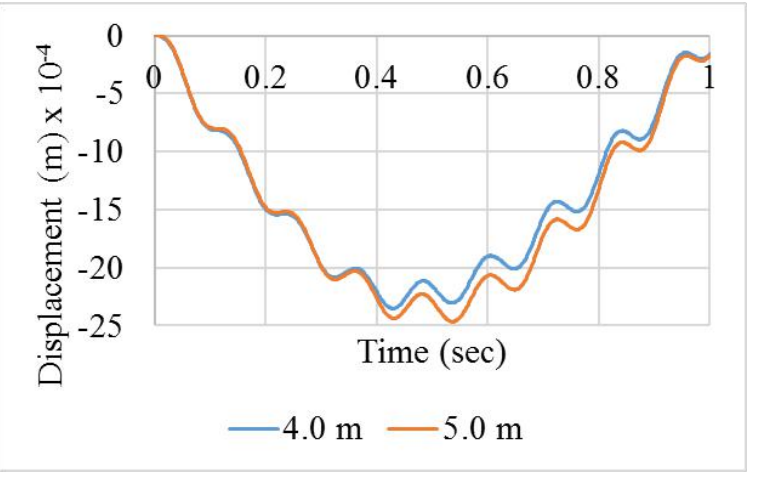

(a)

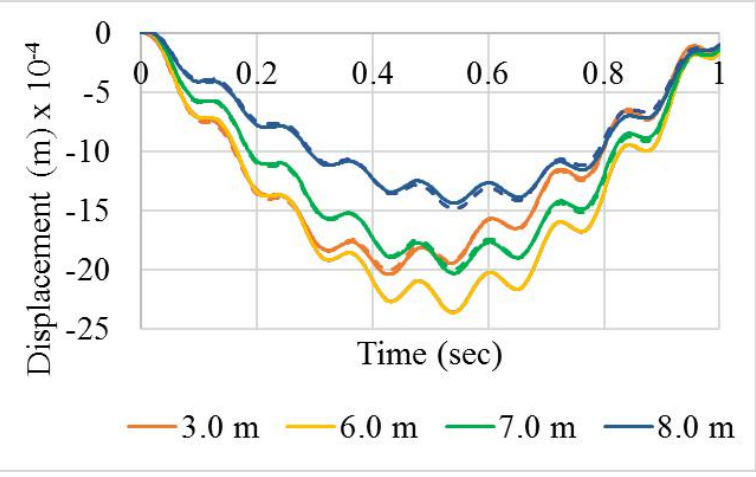

(b)

Figure 2(a); Measured displacement at 4.0m and 5.0m away from the left support, (b) comparison between the estimated displacement (straight line) and the actual displacement (dotted).

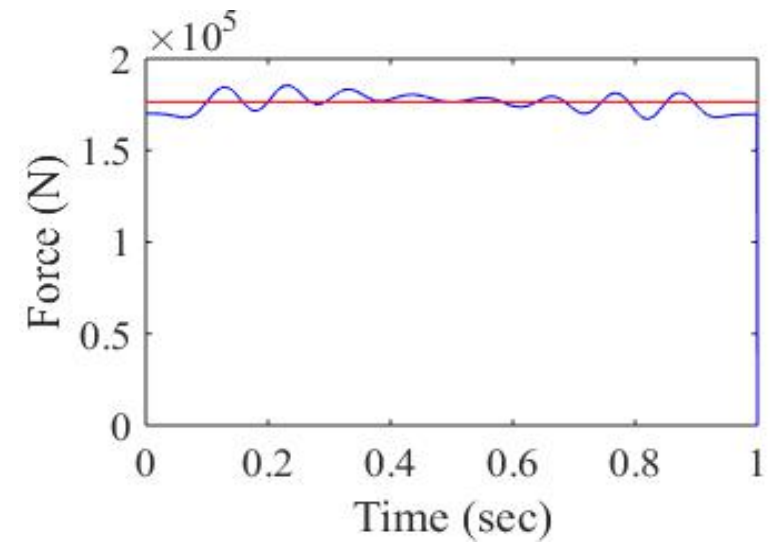

(a)

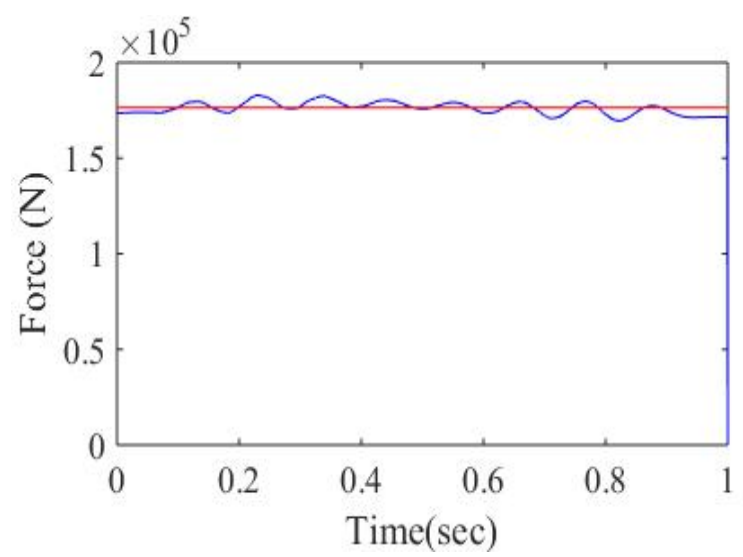

(b)

Figure 3(a); Force history using 2-measured displacement, (b) Force history using 2 -measured + 4-estimated displacement. 


\section{Conclusion}

In conclusion, the pseudoinverse of the mode shape matrix has the ability to estimate the modal response with high accuracy using limited number of measurement. The technique reduces the installation and the instrumentation cost and time of the B-WIM system because it reduced the number of sensors that required to monitor the bridge response. On the same time, it allows for high accurate and cheap loads monitoring by increasing the measurement number.

\section{Acknowledgments}

The authors would like to express their gratitude for the financial support received from the National Science Foundation (NSF-CNS-1645863) for this investigation.

\section{References}

1. ASCE, 2017 Report card for America's infrastructure, 2017.

2. O'Brien EJ, Znidaric A, Dempsey AT. Comparison of two independently developed bridge weigh - in - motion systems. International Journal of Heavy Vehicle Systems 1999;6:147-161.

3. Richardson J, Jones S, Brown A, et al. On the use of bridge weigh-in-motion for overweight truck enforcement. International Journal of Heavy Vehicle Systems 2014; 21: 83-104.

4. Chan THT, O'Connor C. Wheel loads from highway bridge strains: field studies. Journal of Structural Engineering 1990; 116: 1751-1771.

5. Chan TH, Law S, Yung T. Moving force identification using an existing prestressed concrete bridge. Engineering Structures 2000; 22: 1261-1270.

6. Jiang R, Au F, Cheung Y. Identification of masses moving on multi-span beams based on a genetic algorithm. Computers \& Structures 2003; 81: 2137-2148.

7. Law S, Fang Y. Moving force identification: optimal state estimation approach. Journal of Sound and Vibration 2001; 239: 233-254.

8. Pinkaew T. Identification of vehicle axle loads from bridge responses using updated static component technique. Engineering Structures 2006; 28: 1599-1608.

9. Elhattab A, Uddin N, The Implication of Analysis Module on Vehicle Bridge Interaction Modelling, Civil engineering research journal, 2 (2017).

10. Elhattab A, Uddin N, OBrien E. Drive-by bridge damage monitoring using Bridge Displacement Profile Difference. Journal of Civil Structural Health Monitoring 2016; 6: 839-850.

11. ElHattab A, Uddin N, OBrien E. Drive-by bridge damage detection using non-specialized instrumented vehicle. Bridge Structures 2017; 12: 73-84.

12. Elhattab A, Uddin N, OBrien E. Identifying Localized Bridge Damage Using Frequency Domain Decomposition. 26th ASNT Research Symposium 2017; pp. 75-83.

13. Elhattab A, Uddin N, OBrien E. Drive-by Bridge Inspection Using Inverse Dynamics Optimization Algorithm. 26th ASNT Research Symposium 2017; pp. 55-65.

14. Elhattab A, Uddin N, OBrien E. Drive-by Bridge Damage Detection Using Non-specialized Vehicle. 26th ASNT Research Symposium 2017; pp. 43-54.

15. Elhattab A, Uddin N, OBrien E, et al. Field Verification for Drive-by Bridge Monitoring using Non-specialized Inspection Vehicle. 26th ASNT Research Symposium 2017; pp. 66-74.

16. Elhattab AA. Drive-by bridge damage inspection, The University of Alabama at Birmingham, 2015.

17. Tan C, Elhattab A, Uddin N. "Drive-by" bridge frequency-based monitoring utilizing wavelet transform. Journal of Civil Structural Health Monitoring 2017; 7: 615-625.

18. Tan C, Elhattab A, Uddin N. Wavelet Based Damage Assessment and Localization for Bridge Structures. 26th ASNT Research Symposium 2017; pp. 228-240.

19. Winardi E, Elhattab A, Uddin N. Bridge Curvature for Detecting Bridge Damage Location. 26th ASNT Research Symposium 2017; pp. 275-283.

20. González A, Rowley C, OBrien EJ. A general solution to the identification of moving vehicle forces on a bridge. International Journal for Numerical Methods in Engineering 2008; 75: 335-354.

21. Mohammed YM, Uddin N. Bridge Damage Detection using the Inverse Dynamics Optimization Algorithm. 26th ASNT Research Symposium 2017; pp. 175-184.

22. Azam SE, Chatzi E, Papadimitriou C. A dual Kalman filter approach for state estimation via output-only acceleration measurements. Mechanical Systems and Signal Processing 2015; 60: 866-886.

23. Zhi L, Li Q, Fang M. Identification of Wind Loads and Estimation of Structural Responses of Super - Tall Buildings by an Inverse Method. Computer - Aided Civil and Infrastructure Engineering 2016;31: 966-982.

24. Cebon D. Handbook of vehicle-road interaction, ALWAYS 1999.

25. Harris NK, OBrien EJ, González A. Reduction of bridge dynamic amplification through adjustment of vehicle 
suspension damping. Journal of Sound and Vibration 2007; 302: 471-485.

26. El-hattab A, Uddin N, Obrien E. Drive-by bridge damage detection using apparent profile. First International Conference on Advances in Civil Infrastructure and Construction Materials (CISM) 2015; pp. 33-45.

27. Yang YB, Yau J, Yao Z, et al. Vehicle-bridge interaction dynamics: with applications to high-speed railways. World Scientific 2004.

28. González A. Vehicle-bridge dynamic interaction using finite element modelling. Finite element analysis, InTech 2010. 